\title{
Entropy Loading Design for the MIMO-OFDM Visible Light Communication System Using the OCT Precoding Technique
}

\author{
Xinyue Guo, ${ }^{1}$ Haimeng Zhao, ${ }^{1}$ and Wei Wang $\mathbb{C}^{2}$ \\ ${ }^{1}$ Shanghai Key Lab of Modern Optical System, School of Optical-Electrical and Computing Engineering, \\ University of Shanghai for Science and Technology, Shanghai 200093, China \\ ${ }^{2}$ Naval Medical Center of PLA, Shanghai 200433, China \\ Correspondence should be addressed to Wei Wang; wwang_fd@fudan.edu.cn
}

Received 31 July 2020; Accepted 11 September 2020; Published 19 September 2020

Academic Editor: Junmin Liu

Copyright (c) 2020 Xinyue Guo et al. This is an open access article distributed under the Creative Commons Attribution License, which permits unrestricted use, distribution, and reproduction in any medium, provided the original work is properly cited.

In this paper, an orthogonal circulant matrix transform (OCT) precoding technique is proposed to combine with the entropy loading in the multiple-input multiple-output and orthogonal frequency division multiplexing (MIMO-OFDM) visible light communication (VLC) system where the space-time coding (STBC) is chosen for its robustness to the channel correlation. Benefitting from the OCT precoding technique, the uniform signal-to-noise ratio (SNR) among all the subchannels can be achieved. As a result, only one SNR value is required to be fed back, and the same distribution matcher is employed during probabilistic shaping (PS), which means much lower feedback overhead and system complexity than the conventional entropy loading scheme. Experimental results show that the OCT precoding does not cause the system performance loss where the achievable information rate (AIR) of the proposed system is comparable with the conventional system without precoding. With an available bandwidth of $\sim 25 \mathrm{MHz}$, the proposed scheme can realize the AIR of $50.75 \mathrm{Mb} / \mathrm{s}$ at the expense of $0.45 \%$ average forward error correction (FEC) overhead $(\mathrm{OH})$.

\section{Introduction}

Recently, the emergence of "smart home" and the rapid spread of intelligent devices have made a great challenge to the conventional technology of the network. The world is experiencing a profound revolution of access technology called "Anywhere, Anytime" [1]. As a result, new access technologies are urgently required where visible light communication (VLC) using white light-emitting diodes (LEDs) as light sources to transmit the optical signals through the air has received particular attention in both academia and industry [2]. Compared to conventional radio frequency (RF) communication, VLC has numerous advantages such as rich spectrum, safe to human eyes, low power consumption, immune to electromagnetic interference, and so on [3].

High-speed indoor communication is considered as one of the most important applications for VLC systems.
However, the modulation bandwidth of the LED is limited ranging from a few megabytes to tens of gigabytes where the channel response is attenuated exponentially with the frequency increasing [4]. To improve the data rate of the VLC system, numerous techniques have been proposed, among which orthogonal frequency division multiplexing (OFDM) [5] and multiple-input multiple-output (MIMO) [6] are considered especially effective to increase the spectral efficiency for VLC systems. By dividing the channel into several subchannels, OFDM is able to overcome the intersymbol interference (ISI) caused by a nonideal LED frequency response under the condition of high-speed transmission. While MIMO has been proposed to increase the data rate or improve the performance without the extra need of frequency resources by equipping multiple antennas at the transmitter and receiver. Since usually several LEDs are required to support sufficient illumination of the room, it is natural to implement the MIMO technique in VLC systems. 
However, as intensity modulation with direct detection (IM/ DD) is applied in VLC, the value of transmitted signals is real, that is, no phase information can be provided, which leads to a high correlation of the VLC MIMO channel [7]. Consequently, the conventional MIMO scheme based on spatial multiplexing (SMP) cannot be applied successfully in the VLC system, as the bit error rate (BER) performance decreases sharply as soon as the channel is highly correlated. Compared with SMP, MIMO scheme based on transmit diversity has been proved to be robust to the channel correlation, so it is more suitable for VLC systems [8]. However, as the transmit diversity scheme mainly enjoys the advantage of diversity gains, it lacks multiplexing gains. So, how to improve the data rate remains to be studied.

Adaptive modulation implemented by bit allocation has been proposed to improve the data rate close to the Shannon capacity in MIMO-OFDM VLC systems [9]. Through adaptive modulation, it has been shown that data rate can be increased without sacrificing the BER performance. Furthermore, some papers mentioned that the bit allocation algorithm can only assign discrete bits to each subchannel, leading to a gap from the channel capacity. Consequently, an entropy loading scheme has been proposed to narrow the gap from the channel capacity [10]. By introducing the probabilistic shaping (PS) quadrature amplitude modulation (QAM) technique $[11,12]$, the uniformly distributed signal source is transformed to Gaussian distribution, and the continuous entropy can be realized in the entropy loading scheme. Moreover, the effect of the nonlinear distortion induced by the LED can also be reduced for the modulated signals with a higher power tend to appear with lower probabilities according to the idea of the PS technique. However, the main disadvantage of the entropy loading scheme is that a large feedback is required, and the complexity is high. Since the frequency response of the LED is attenuated exponentially with the frequency increasing, the signal-to-noise ratios (SNRs) on each subchannel are different. Thus, it is necessary to feedback all the SNRs from the receiver to the transmitter. Moreover, different distribution matchers are required to generate signal sources with different probabilistic distribution for each subchannel when employing PS.

To deal with this problem, a precoding scheme based on orthogonal circulant matrix transform (OCT) $[13,14]$ is proposed to be combined with the entropy loading in the MIMO-OFDM VLC systems in this paper. Thanks to the equalization effect of OCT, SNRs are almost the same on all the subchannels, which means that only one SNR value is needed to be fed back. Meanwhile, only one distribution matcher is required benefiting from the SNR equalization effect on all subchannels, which maximally reduces the complexity of the entropy loading scheme. Experimental results show that achievable information rate (AIR) of the proposed system is comparable with the conventional system without precoding. With an available bandwidth of $\sim 25 \mathrm{MHz}$, the proposed scheme can realize the AIR of $50.75 \mathrm{Mb} / \mathrm{s}$ at the expense of $0.45 \%$ average forward error correction (FEC) overhead $(\mathrm{OH})$.

\section{Operation Principle}

Under the condition of limited modulation bandwidth, the entropy loading transmission scheme based on PS can effectively narrow the gap with the Shannon limit. In the entropy loading scheme, channel capacity is approached by a Gaussian source where constellation points of M-QAM are assigned different probabilities by a probabilistic distribution matcher. Obviously, SNR has to be known at the transmitter during the calculation of the channel capacity. However, due to the frequency selective characteristics of the LED, the SNRs of different subchannels are also different, which indicate that lots of information have to be fed back. Moreover, different kinds of distribution matchers are required to implement PS on each subchannel. In order to reduce the feedback and the system complexity, we proposed an OCT precoding scheme to combine with the entropy loading. OCT precoding was first proposed $[13,14]$ where preequalization is realized without feeding back channel state information. Therefore, we introduce OCT precoding in the entropy loading scheme to equalize the SNRs uniformly over all subchannels. In this way, the feedback overhead and the system complexity can be greatly reduced, that is, only one SNR is required to be fed back, and data source on different subchannels can use the same distribution matcher.

Without the loss of generality, consider a MIMO-OFDM VLC system configured with two LEDs as transmitters (TX) and two photoelectric detectors (PDs) as receivers (RX). In our scheme, OCT precoding is jointly employed over all subchannels where the MIMO channel can be equivalent to two decorrelated channels by using space-time block coding (STBC).

According to the Hong et al. [14], the OCT matrix, which is constructed by the Zad-off Chu (ZC) sequence [15], can be expressed by

$$
[\mathbf{F}]_{(2 K) \times(2 K)}=\frac{1}{\sqrt{2 K}}\left[\begin{array}{cccc}
f_{1} & f_{2} & \cdots & f_{2 K} \\
f_{2 K} & f_{1} & \cdots & f_{2 K-1} \\
\cdots & \cdots & \cdots & \cdots \\
f_{2} & f_{3} & \cdots & f_{1}
\end{array}\right],
$$

where $K$ denotes the subchannel number for each decorrelated, channel and $f_{l}(1 \leq l \leq 2 K)$ is the corresponding element of the $\mathrm{ZC}$ sequence with a length of $2 K$.

Then, the precoding signals can be given by

$$
\overline{\mathbf{X}}=\left[\begin{array}{l}
\overline{\mathbf{X}}_{1} \\
\overline{\mathbf{X}}_{2}
\end{array}\right]=\mathbf{F} \cdot\left[\begin{array}{l}
\mathbf{X}_{1} \\
\mathbf{X}_{2}
\end{array}\right]
$$

where $\mathbf{X}_{i}=\left[X_{i}(1), X_{i}(2), \ldots, X_{i}(K)\right]^{T}$ denotes the vector of the signals from the $i^{\text {th }}$ transmitted data stream $(i=1,2)$. As shown, signals on the frequency domain are multiplied by an orthogonal circulant matrix through OCT precoding; then, the information on each subchannel is spread across all subchannels to achieve the frequency diversity. 
Generalized mutual information (GMI), normalized GMI (NGMI), and FEC OH are chosen as the main figures of merit (FOM) to evaluate the performance of the system [16]. GMI quantifies the maximum number of information bits per transmitted symbol after ideal decoding, and the GMI can be obtained by log-likelihood ratios (LLRs) based on Monte Carlo simulations [17].

Assume that the discrete channel input $\mathbf{X}^{\prime}=\left\{X_{1}^{\prime}, X_{2}^{\prime}, \ldots, X_{N}^{\prime}\right\}$ is independent and identically distributed, and the $\mathbf{Y}^{\prime}$ represents the corresponding channel output, in which $X_{i}^{\prime} \in \chi, \chi=\left\{x_{1}, x_{2}, \ldots, x_{M}\right\}$ and the symbol $\mathbf{X}^{\prime}$ consists of $m$ bits levels:

$$
\begin{aligned}
& \mathrm{GMI} \approx-\sum_{x \in \chi} P_{\mathbf{X}^{\prime}}(x) \log _{2} P_{\mathbf{X}^{\prime}}(x) \\
&+\frac{1}{N} \sum_{k=1}^{N} \sum_{i=1}^{m} \log _{2} \frac{\sum_{x \in \chi_{b_{k, i}}} q_{\mathbf{Y}^{\prime}} \mid \mathbf{X}^{\prime}}{\sum_{x \in \chi} q_{\mathbf{Y}^{\prime}} \mid \mathbf{X}^{\prime}}\left(y_{k} \mid x\right) P_{\mathbf{X}^{\prime}}(x) \\
& \mathbf{X}_{\mathbf{X}^{\prime}}(x)
\end{aligned}
$$

where $P_{\mathbf{X}^{\prime}}(x)$ denotes the corresponding distribution probability mass function, $q_{\mathbf{Y}^{\prime} \mid \mathbf{X}^{\prime}}\left(y_{k} \mid x\right)$ is given by $q_{\mathbf{X}^{\prime} \mid \mathbf{X}^{\prime}}\left(y_{k} \mid x\right)=\left(1 / \sqrt{2 \pi \sigma^{2}}\right) \cdot e^{\left(\left(-\left|y_{k}-x\right|^{2}\right) / 2 \sigma^{2}\right)}, b_{k, i} \in\{0,1\}$ is the $i^{\text {th }}$ bit of the $k^{\text {th }}$ transmit symbol, and the $\chi_{b_{k, i}}$ is the set of constellation symbols whose $i^{\text {th }}$ bit value is $b_{k, i}$.

After the GMI is estimated, the NGMI of the PS-MQAM source [11], which is used to evaluate the BER performance after the forward error correction (FEC), can be obtained by

$$
\mathrm{NGMI}=1-\frac{H\left(P_{\mathbf{X}^{\prime}}\right)-\mathrm{GMI}}{m},
$$

where $H\left(P_{\mathbf{X}^{\prime}}\right)$ is the entropy of the constellation.

Once the NGMI is calculated, the FEC OH can be determined, which is a threshold for error-free post-FEC results $[18,19]$. After removing the $\mathrm{OH}$, the AIR of the individual subcarrier can be calculated by Equation (5). And the average of them is the total AIR of the whole system:

$$
\mathrm{AIR}=B \cdot \mathrm{GMI} \cdot(1-\mathrm{OH}),
$$

where $B$ denotes the baud rate, and the $\mathrm{OH}$ is given by $\mathrm{OH}=((1-\mathrm{NGMI}) / \mathrm{NGMI})$.

\section{System Configuration and Results}

In this session, an experimental demonstration is set up to study the performance to prove the excellence of the proposed system. In Figure 1, the block diagram and the experimental setup of the proposed VLC system are illustrated. The distance between the transmitter side and the receiver side is $0.8 \mathrm{~m}$. At the transmitter, the source distribution is determined by the channel capacity, which is calculated according to the feedback information of SNR. Then, the certain distributed source is PS by the distribution matcher. After QAM modulation and serial-to-parallel (S/P) conversion, the OCT precoding is jointly implemented over all subchannels. Finally, STBC and OFDM are applied to generate the transmitted signals. Noted that signals transmitted by the VLC system must be real-valued, so only half of the subcarriers are used to transmit signals during OFDM modulation, and the other half is used to transmit complex conjugate signals of the above signals. Besides, among the half of the subcarriers, there are six subcarriers (from the 1st subcarrier to the 6th subcarrier) of the low frequency suffering from the severe fading SNR, which may degrade the system performance. As a result, zero-padding is used.

In the experiment, the arbitrary function generator (AFG, Tektronix AFG3252C) is used to generate the transmitted signals at $100 \mathrm{MSa} / \mathrm{s}$. Meanwhile, direct current (DC) offset supplied by AFG is set to ensure that the electrical OFDM signals are positive. Then, the mixed signals are transmitted in the form of optical power by the two commercially available LEDs (Cree XLamp XP-E) radiating red light, whose center wavelength is $620 \mathrm{~nm}$ and maximum power is $1 \mathrm{~W}$. Because the LED is a point light source, a reflection cup with $60^{\circ}$ is used to concentrate the light. At the receiver, PDs (Hamamatsu C12702-11, 0.42 A/W responsivity at $620 \mathrm{~nm}$ ) with $1 \mathrm{~mm}^{2}$ active area and about $100 \mathrm{MHz}$ bandwidth are used. Then, optical signals entering the PDs are converted into electrical signals and amplified by an electrical amplifier (EA) circuit. Finally, electrical signals are collected by a real-time oscilloscope (OSC, Tektronix MDO4104 C) with a sampling rate of $100 \mathrm{MSa} / \mathrm{s}$. The system parameters of our experiments are listed in Table 1.

The signal processing at the receiver is the inverse process of the transmitter. After frame synchronization, the signals are first converted to the frequency domain by OFDM demodulation. Then, the channel and the noise variance are estimated with the help of the preamble for the sake of signal decoding and the SNR estimation. Since the SNR values of different subcarriers and different receivers are slightly different due to the random noise, the SNR value used for feedback is calculated by averaging the SNR values over different subcarriers and different receivers, that is, only one SNR value is required to be fed back from the receiver to the transmitter. Here, we assume that the transmitter can obtain the exact SNR information. Through STBC decoding and OCT decoding, finally, the binary bit sequence is recovered after M-QAM demapping.

To make the comparison fair, the same QAM order is used for the conventional entropy loading scheme and the proposed OCT precoding based on the entropy loading scheme. In Figure 2, the SNR results of the conventional and proposed entropy loading schemes for two receivers are given. As shown, there are slight differences among the SNR values of different receivers because of the random noise. As a result, the SNR curves of two receivers are similar with the same trend. As displayed, the flat SNR curve across the spectral can be obtained in the proposed scheme owing to the application of the OCT precoding. While for the conventional scheme, the SNR fluctuations over subcarriers are more than $10 \mathrm{~dB}$ because of the attenuation of channel frequency response.

In Figure 3, the experimental results are illustrated when 32-QAM is employed in the system. In the experiment, the BERs of the conventional scheme and the proposed scheme are $8.04 \times 10^{-4}$ and $7.15 \times 10^{-4}$, respectively, which are both below the $7 \%$ the preforward error correction (pre-FEC) 


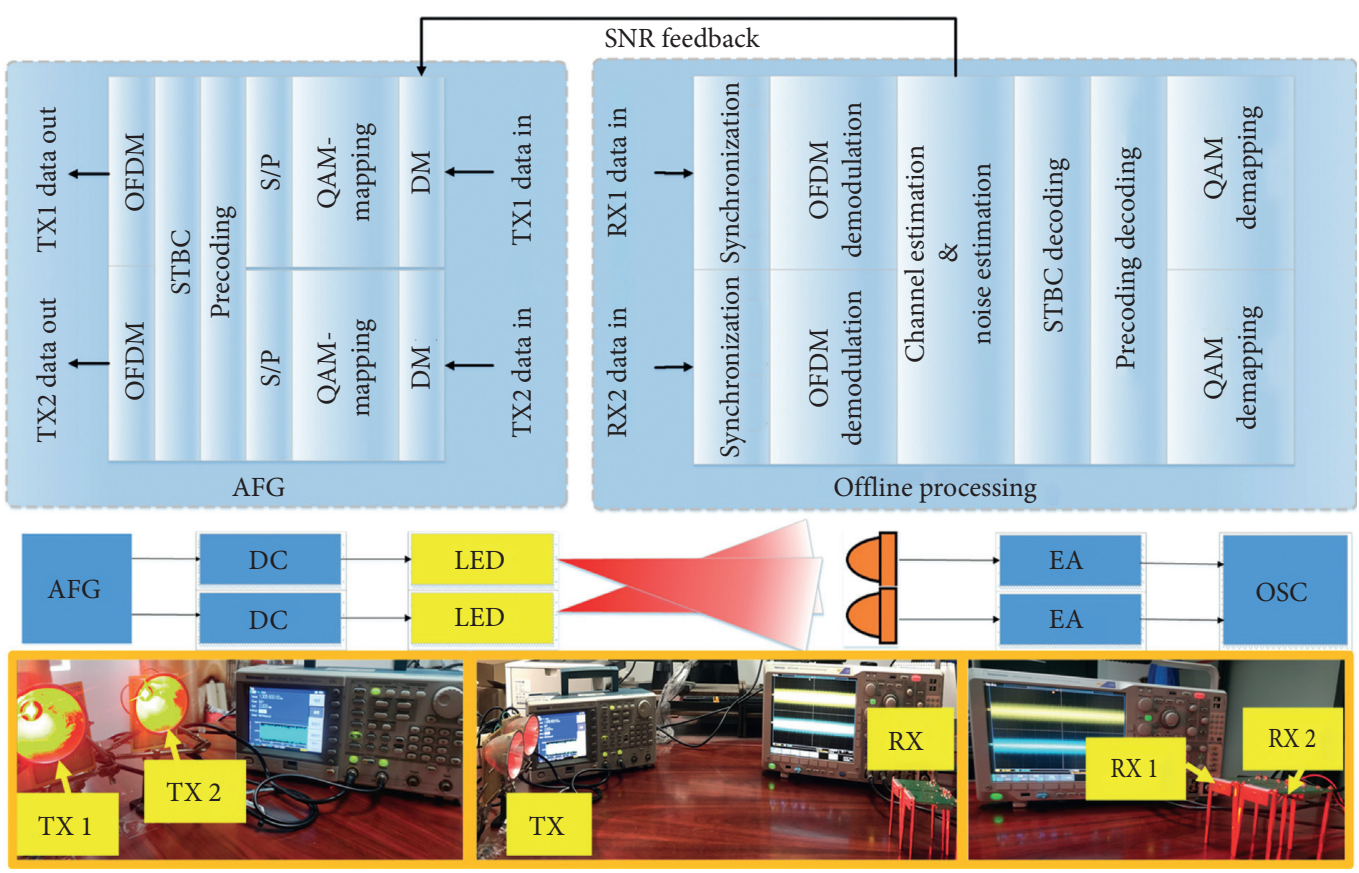

FIgURE 1: Experimental setup of the proposed MIMO-OFDM VLC system.

TABLE 1: System parameters.

\begin{tabular}{lc}
\hline Parameters & Values \\
\hline Bandwidth & $0-25 \mathrm{MHz}$ \\
Subcarrier number & 256 \\
Upsampling rate & 4 \\
Transmission distance & $0.8 \mathrm{~m}$ \\
DC offset & $2 \mathrm{~V}$ \\
Vpp & $2 \mathrm{~V}$ \\
\hline
\end{tabular}

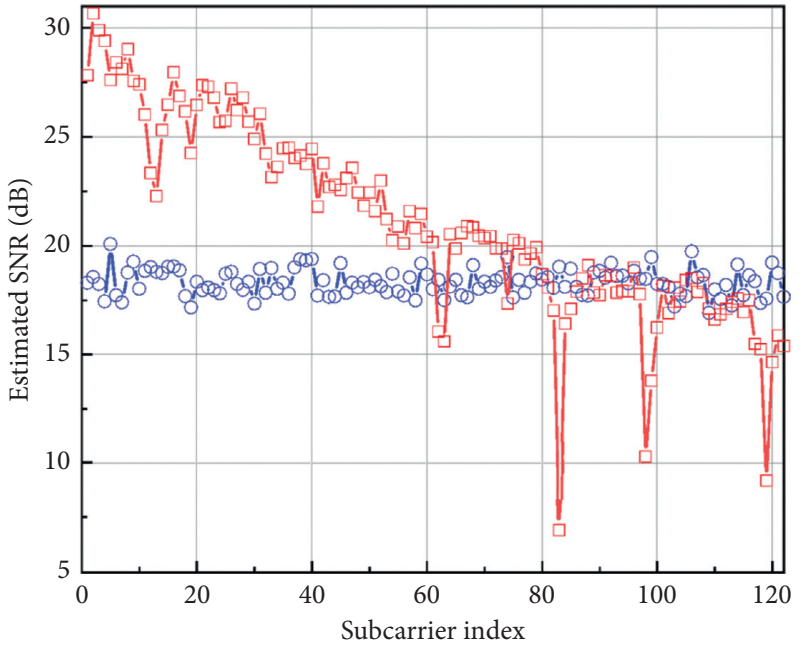

-o- Proposed scheme (receiver 1)

$-\square-$ Conventional scheme (receiver 1)

(a)

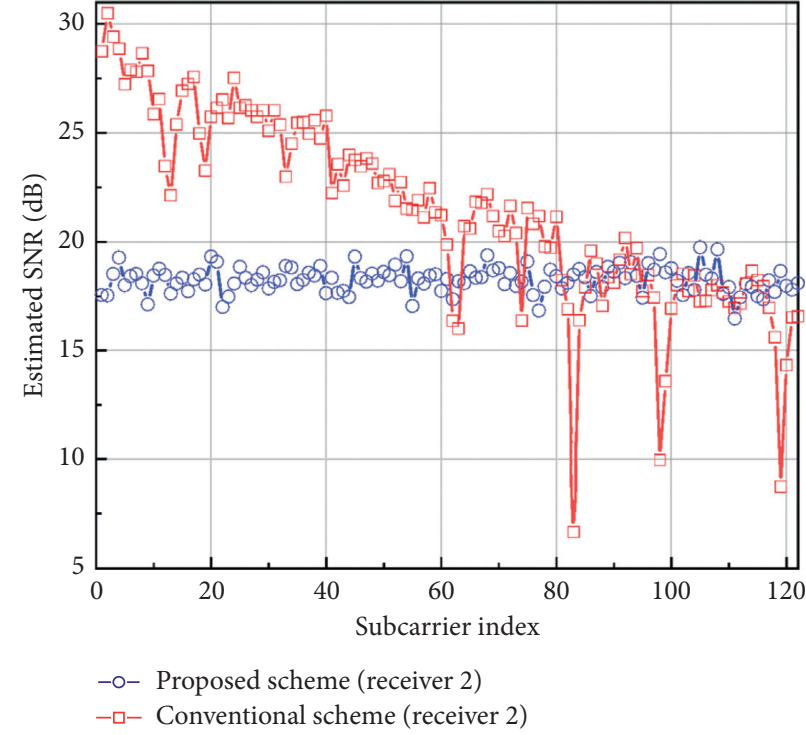

(b)

FIGURE 2: Estimated SNR as a function of subcarrier index. 


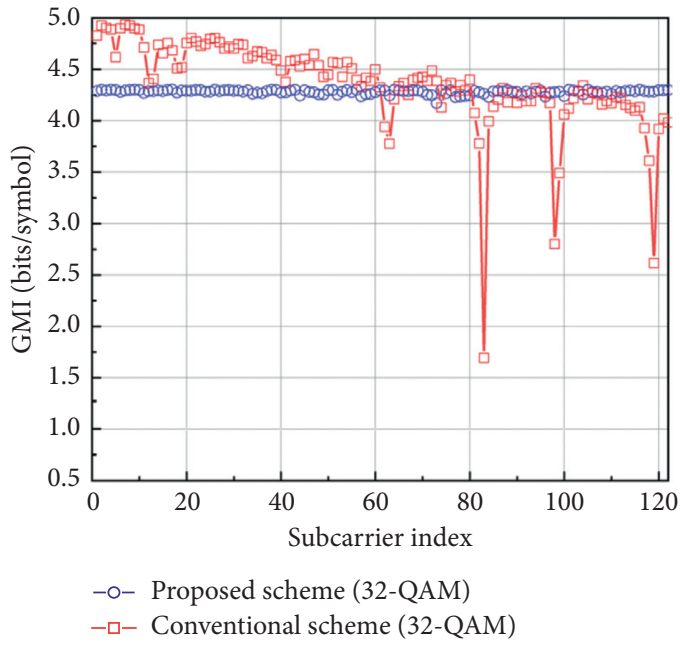

(a)

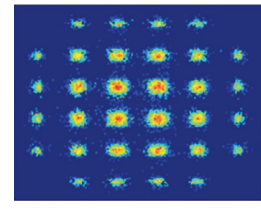

(c)

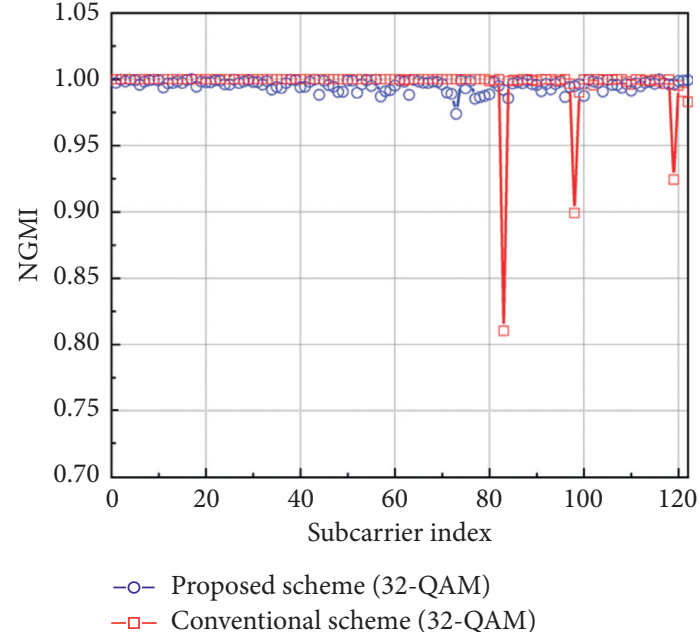

(b)

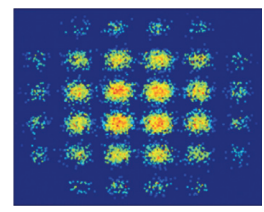

(d)

Figure 3: Experimental verification when the modulation order is 32-QAM: (a) GMI as a function of subcarrier index, (b) NGMI as a function of subcarrier index, (c) corresponding constellation density of the scheme without precoding, and (d) corresponding constellation density of the proposed scheme with OCT precoding.

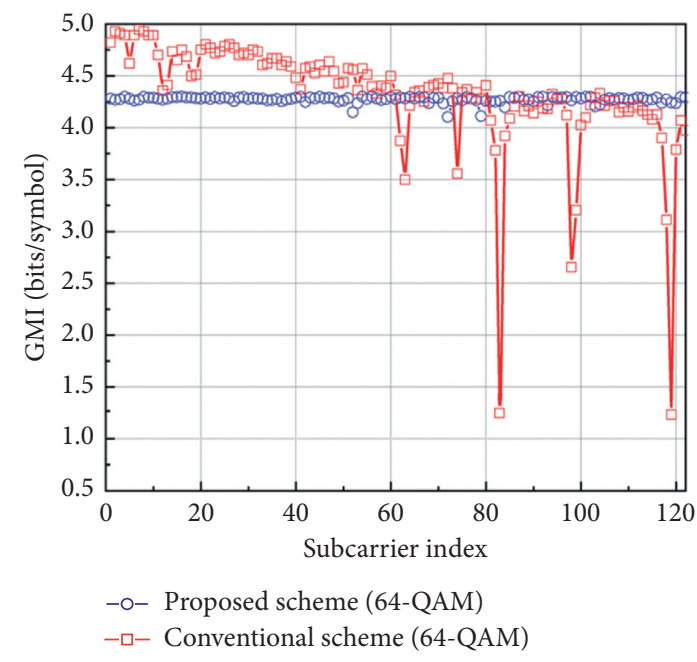

(a)

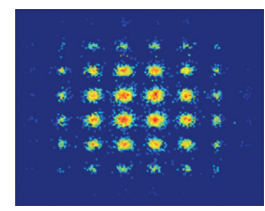

(c)

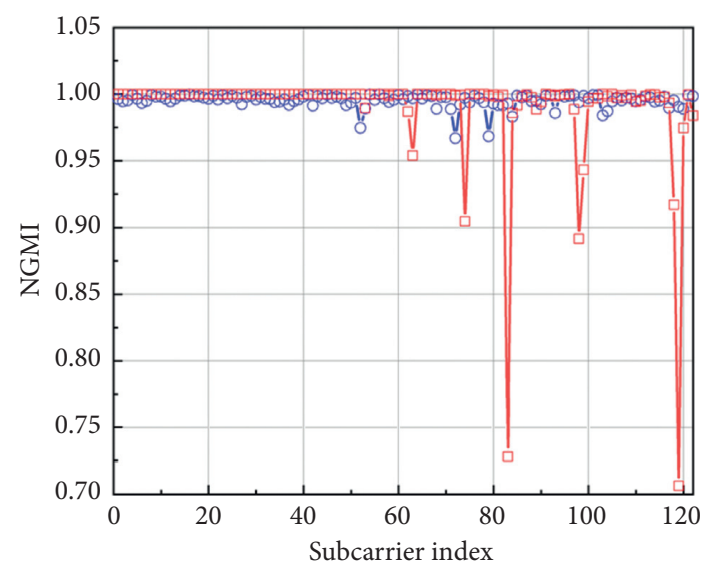

-o- Proposed scheme (64-QAM)

$-\square-$ Conventional scheme (64-QAM)

(b)

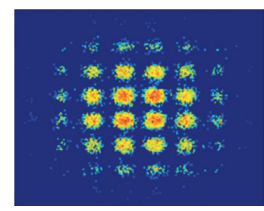

(d)

FIgURE 4: Experimental verification when modulation order is 64-QAM: (a) GMI as a function of subcarrier index, (b) NGMI as a function of subcarrier index, (c) corresponding constellation density of the scheme without precoding, and (d) corresponding constellation density of the proposed scheme with OCT precoding. 
TABLE 2: AIR performance comparison in the experiment.

\begin{tabular}{lcccc}
\hline & \multicolumn{2}{c}{ Condition 1 } & (32-QAM) & \multicolumn{2}{c}{ Condition 2 (64-QAM) } \\
& Conventional scheme & Proposed scheme & Conventional scheme & Proposed scheme \\
\hline AIR $(\mathrm{Mb} / \mathrm{s})$ & 51.88 & 50.75 & 51.25 & 50.65 \\
Average FEC OH & $0.42 \%$ & $0.45 \%$ & $1.15 \%$ & $0.48 \%$ \\
\hline
\end{tabular}

threshold of $3.8 \times 10^{-3}$. As shown in Figure 3(a), the results of the GMI curves agree with the estimated SNR curves where the GMI of the conventional scheme decreases sharply because of the attenuation of channel frequency response, while the GMI of the proposed scheme is uniform across all subcarriers, thanks to the OCT precoding. In Figure 3(b), the results of NGMI are given. It can be seen that NGMI of the conventional scheme is close to 1 on most of the subcarriers. However, the severe fading NGMI of several subcarriers may degrade the total performance. While the NGMI curve of the proposed scheme with OCT precoding is flat just as the GMI curve in Figure 3(a). Figures 3(c) and $3(d)$ represent the constellation density of two schemes. Because of the PS technique, the constellation points with lower energy are assigned higher probabilities. In this way, the nonlinear distortion induced by LEDs can also be reduced. Furthermore, the system performance is studied when 64-QAM is used, and the experimental results are shown in Figures 4(a)-4(d). The GMI and NGMI curves are similar to the case of 32-QAM. The BERs of the conventional scheme and the proposed scheme are 0.0021 and $5.12 \times 10^{-4}$, both of which are a little higher than the results of the 32QAM case. The results indicate that the nonlinearity of the LED would impact the BER performance as the modulation order grows.

In Table 2, the results of AIR performance comparison are listed. When the modulation order is equal to 32 , the total AIRs of the conventional and proposed systems are $51.88 \mathrm{Mb} / \mathrm{s}$ and $50.75 \mathrm{Mb} / \mathrm{s}$, respectively, and the average FEC OHs are $0.42 \%$ and $0.45 \%$. The results indicate that both systems have nearly the same transmission rate and the $\mathrm{OH}$, which proves that the OCT precoding does not cause the performance loss. When 64-QAM is applied to the system, the total AIRs of the conventional and proposed systems are $51.25 \mathrm{Mb} / \mathrm{s}$ and $50.65 \mathrm{Mb} / \mathrm{s}$, with the average FEC OHs equal to $1.15 \%$ and $0.48 \%$, respectively. Compared with the 32 QAM-based system, the AIRs decrease slightly because more nonlinear distortion tends to occur in the high-order modulation system. Consequently, more $\mathrm{OHs}$ are required especially in the conventional system without precoding.

\section{Conclusion}

In this paper, the OCT precoding scheme is proposed to combine with the entropy loading in MIMO-OFDM VLC systems to reduce the system feedback and complexity significantly without performance loss. Through OCT precoding, SNRs among different subchannels are equalized uniformly owing to the advantage of the frequency diversity. As a result, only one SNR value is required to be fed back to the transmitter, and only one distribution matcher is needed in the process of PS, leading to a much lower feedback overhead and system complexity. Finally, an experimental demonstration is set up to evaluate the performance of the proposed system. The experimental results confirm that the AIR of the proposed system is similar to the value of the conventional system without precoding. With an available bandwidth of $\sim 25 \mathrm{MHz}$, the proposed scheme can experimentally achieve the AIR of $50.75 \mathrm{Mb} / \mathrm{s}$ at the expense of $0.45 \%$ average $\mathrm{FEC} \mathrm{OH}$.

\section{Data Availability}

The data used to support the findings of this study are available from the corresponding author upon request.

\section{Conflicts of Interest}

The authors declare that they have no conflicts of interest.

\section{Acknowledgments}

This work was supported by the Foundation of China (No. HJ20172B0202718K503).

\section{References}

[1] A. A. Dowhuszko, M. C. Ilter, and J. Hämäläinen, "Visible light communication system in presence of indirect lighting and illumination constraints," in Proceedings of the ICC 20202020 IEEE International Conference on Communications (ICC), pp. 1-6, Dublin, Ireland, June 2020.

[2] S. Keshari, R. Gupta, and R. Bhargava, "Realization of visible light communication approach with power LED and solar panel," in Proceedings of the 2019 International Conference on Computing, Power and Communication Technologies (GUCON), pp. 953-958, New Delhi, India, 2019.

[3] A.-M. Cailean and M. Dimian, "Current challenges for visible light communications usage in vehicle applications: a survey," IEEE Communications Surveys \& Tutorials, vol. 19, no. 4, pp. 2681-2703, 2017.

[4] P. A. Bhaskar, B. K. Jeemon, and S. Dhanya, "Performance analysis of LDPC MIMO-OFDM system for frequency selective fading channels," in Proceedings of the 2017 International Conference on Energy, Communication, Data Analytics and Soft Computing (ICECDS), pp. 1802-1806, Chennai, India, August 2017.

[5] X. Chen, Z. Feng, M. Tang, S. Fu, and D. Liu, "Performance enhanced DDO-OFDM system with adaptively partitioned precoding and single sideband modulation," Optics Express, vol. 25, no. 19, pp. 23093-23108, 2017.

[6] S. Wei, "Simulation and analysis of the impact of MIMO system on communication quality," in Proceedings of the 2020 International Wireless Communications and Mobile Computing (IWCMC), pp. 341-344, Limassol, Cyprus, 2020. 
[7] M. Alhalabi, F. I. El-Nahal, and N. Taşpinar, "Comparison of different modulation techniques for optical OFDM Intensity Modulation and Direct Detection IM/DD system," in Proceedings of the IEEE 7th Palestinian International Conference on Electrical and Computer Engineering (PICECE), pp. 1-4, Gaza, Palestine, March 2019.

[8] M.-T. Le, T.-D. Nguyen, X.-N. Tran, and V.-D. Ngo, "On the combination of double space time transmit diversity with spatial modulation," IEEE Transactions on Wireless Communications, vol. 17, no. 1, pp. 170-181, 2018.

[9] Y. Iraqi and A. Al-Dweik, "Adaptive bit loading with reduced computational time and complexity for multicarrier wireless communications," IEEE Transactions on Aerospace and Electronic Systems, vol. 56, no. 3, pp. 2497-2506, 2020.

[10] D. Che and W. Shieh, "Entropy-loading: multi-carrier constellation-shaping for colored-SNR optical channels," in Proceedings of the Optical Fiber Communications Conference and Exhibition (OFC), pp. 1-3, Los Angeles, CA, USA, March 2017.

[11] T. Fehenberger, A. Alvarado, G. Böcherer, and N. Hanik, "On probabilistic shaping of quadrature amplitude modulation for the nonlinear fiber channel," Journal of Lightwave Technology, vol. 34, no. 21, pp. 5063-5073, 2016.

[12] G. Böcherer, P. Schulte, and F. Steiner, "Probabilistic shaping and forward error correction for fiber-optic communication systems," Journal of Lightwave Technology, vol. 37, no. 2, pp. 230-244, 2019.

[13] Y. Hong, X. Guan, L. Chen, and J. Zhao, "Experimental demonstration of an OCT-based precoding scheme for visible light communications," in Proceedings of the Optical Fiber Communications Conference and Exhibition (OFC), pp. 1-3, Anaheim, CA, USA, March 2016.

[14] Y. Hong, L.-K. Chen, and J. Zhao, "Performance-enhanced gigabit/s MIMO-OFDM visible light communications using CSI-free/dependent precoding techniques," Optics Express, vol. 27, no. 9, pp. 12806-12816, 2019.

[15] R. Heimiller, "Phase shift pulse codes with good periodic correlation properties," IEEE Transactions on Information Theory, vol. 7, no. 4, pp. 254-257, 1961.

[16] C. E. Shannon, "A mathematical theory of communication," Bell System Technical Journal, vol. 27, no. 3, pp. 379-423, 1948.

[17] Z. He, W. Liu, C. Xie et al., "Achievable information rate enhancement of visible light communication using probabilistically shaped OFDM modulation," in Proceedings of the Optical Fiber Communications Conference and Exposition (OFC), pp. 1-3, San Diego, CA, USA, March 2018.

[18] J. Cho, L. Schmalen, and P. J. Winzer, "Normalized generalized mutual information as a forward error correction threshold for probabilistically shaped QAM," in Proceedings of European Conference on Optical Communication, pp. 1-3, Göteborg, Sweden, September 2017.

[19] T. Yoshida, A. Alvarado, M. Karlsson, and E. Agrell, "PostFEC BER benchmarking for bit-interleaved coded modulation with probabilistic shaping," Journal of Lightwave Technology, vol. 38, no. 16, pp. 4292-4306, 2020. 